\title{
Mesoscopic Modelling of 2-CN-PPV/PPV Polymer LED
}

\author{
Hélder M. C. Barbosa, Marta M. D. Ramos \\ Departamento de Física, Universidade do Minho \\ Campus de Gualtar, 4710-057 Braga, Portugal
}

\begin{abstract}
Although optoelectronic devices made of polymers are very attractive ones (low cost, easy to make), problems related to charge transport, exciton quenching, among others, can be an obstacle for their performance. The use of heterojunctions made of two polymers can be a strategy for improving the efficiency of polymer light emitting diodes (PLEDs) at low bias. Here we present a theoretical study of the influence of bilayer structure in a PLED made of PPV and 2-CN-PPV, by adopting a mesoscopic approach. Our results show that the presence of the polymer/polymer interface improves charge injection and leads to a confinement of charges near it, which will increase the number recombination events in the middle of the device compared to the equivalent single-layer PLEDs.
\end{abstract}

\section{Introduction}

Since the first report of the use of conjugated polymers as an active layer in LEDs [1], many strategies have been implemented to improve their efficiency and to solve problems related to charge injection, transport and recombination [2]. One of the strategies is to use two layers of different semi-conducting polymers between the electrodes to control charge injection rates, by reducing the potential barriers at electrode/polymer interface, and to improve the transport of holes and electrons through the polymer layer where they were injected. Therefore, due to the different properties of the polymers used, both charge carriers will be confined at the polymer/polymer interface, and for this reason the number of charges that undergoes recombination close to it increases [3]. To improve hole injection from the anode, a hole transport layer (HTL) made of a polymer with low ionization potential is used. This reduces the zero-field barrier height at the anode/polymer interface and increases hole injection. At the same time, by using a polymer that preferentially allows hole mobility, holes will move easily to regions far way from the anode, thus decreasing the number of recombinations near that electrode and preventing exciton quenching. On the other hand, the electron injection at the cathode/polymer 
interface is improved by using an electron transport layer (ETL) made of a polymer that has high electron affinity. The deposition of a metallic cathode on the polymer layer with high electron affinity reduces the zero-field barrier height for electron injection at the cathode/polymer interface, and at the same time improves electron transport towards the opposite electrode. The presence of a polymer/polymer interface in the middle of the device will confine holes and electrons near the interface and in this way will allow an increase in the number of charges that undergo recombination on that region. This strategy seems to improve PLEDs efficiency relative to single polymer layer devices. Although the role of the interface in improving PLED efficiency is established, the way in which charge is confined or the influence of the energetic disorder in the interface, due to the use of polymers with different properties, is far way from being understood. The aim of the present work is to use mesoscopic modelling to study the influence of a bilayer structure on bipolar charge injection, transport, trapping and recombination in PLED as compared with a single layer device based on the same polymer materials.

\section{Model and Simulation Details}

Layers of conjugated polymers are known to have a "spaghetti structure" with chains having several conjugated segments linked by kinks or twists [4]. As a result these polymers can be seen as a disordered system of localized energetic sites where charge injection and transport is temperature activated, in a process known as hopping $[5,6]$. On the other hand, quantum mechanical calculations show that charge mobility along a polymer strand (intra-molecular mobility) is quite different from charge mobility between different polymer strands (inter-molecular mobility) [7]. When a charge is injected into a polymer chain it will stay in the middle of it. But, if the electric field along the polymer molecular axis it is above a certain threshold, which will depend on charge sign, the charge can move to the chain extremity favoured by the field. This process is faster that charge hopping between strands and can play an important role in charge percolation. Since the microstructure of the polymer layer depends on the experimental conditions in which it is prepared, it seems obvious that this will affect both intra-molecular and inter-molecular charge mobility. By considering all of these aspects, we adopt a mesoscopic approach to construct a model where we can study the influence of the polymer morphology, the molecular properties, the electrode work function and even the polymer bulk structure, in charge injection, transport and recombination. 
Since most of these devices are made by the deposition of the polymer thin film on a substrate, by spin-coating, polymer strands should have their molecular axis oriented parallel to the electrode surfaces [8]. To study the behaviour of a bilayer structure in the functioning of a PLED as compared with a single layer structure, three different networks where made using two different polymer materials. By using the same strategy as reported before [9], the three polymer networks of $100 \mathrm{~nm}$ thickness where built between two planar electrodes by stiff-chain segments with a Gaussian length distribution with a mean value of 5 monomers and their axis oriented parallel to the electrode surfaces. One of the networks is made of poly(para-phenylenevinylene) (PPV), another of poly(2-cyano-paraphenylenevinylene) (2-CN-PPV), and the third one is a bilayer of PPV and 2-CN-PPV each with 50 $\mathrm{nm}$ thickness. The cathode and the anode are in contact with 2-CN-PPV and PPV surfaces, respectively.

In our model charge injection into the polymer and inter-molecular charge transport is achieved by considering the hopping probability between the electrodes and the polymer strands, and between neighbouring strands. The injected charge (electrons and holes) percolates through the polymer layer where it can find a charge of opposite sign and undergo recombination, giving rise to both singlet and triplet exciton formation, can be collected by the electrode opposite to the one where the injection occurs or be stored in the polymer network. The hopping probability between two sites depends on the potential barrier between them, the electric field orientation along the jump direction and the jump distance. To calculate the potential barrier between two sites of the polymer network we have to consider the molecular properties of the polymer strands (e.g. ionization potential and electron affinity) where the sites are localized, or the work function of the electrode, when one of the sites is located at the electrode, and the electric potential. The electric field along the jump distance and the electric potential between both sites are calculated by considering the applied electric field, the space charge distribution and the electrode polarization.

To study the bipolar charge evolution in the three polymer networks, we consider that both electrodes are perfect ones (i.e. with no impurities, no roughness, etc) and the imposed work functions of the electrodes are such that the anode/PPV and cathode/2-CN-PPV zero-field barrier height is approximately $3.2 \times 10^{-20} \mathrm{~J}$, working like Ohmic contacts [6]. The zero-field barrier height is calculated considering the difference between the electrode work function and the average ionization potential of the strands (for hole injection) or average electron affinity of the strands (for electron injection). The reason for this lies on the fact that the properties of the polymer strands depend of the length of the 
strand, and since we have a Gaussian distribution of lengths, this seems to be the most correct procedure.

\section{Results and Discussion}

In order to study the influence of the PPV/2-CN-PPV bilayer structure on charge injection, transport, trapping and recombination in the modelled PLED, compared to PPV and 2-CN-PPV single-layer structures, the applied electric field is varied from $1 \mathrm{MV} / \mathrm{cm}$ to $5 \mathrm{MV} / \mathrm{cm}$. For all the polymer structures studied, an increase in the applied electric field gives rise to an increase in the total number of injected charges, but for the same applied electric field the bilayer structure and the 2-CN-PPV single-layer have the same injection efficiency, which is higher than the one obtained for the PPV layer (see Fig. 1).

For a particular applied electric field, charge transport and injection depend, among other factors, on the potential barrier between the hopping sites. For single-layer LEDs, the zero-field barrier heights of cathode/PPV and anode/2-CN-PPV interfaces are $1.06 \times 10^{-19} \mathrm{~J}$ and $6.88 \times 10^{-20} \mathrm{~J}$ respectively, which makes them at the beginning non-ohmic contacts. These values indicate that the injection of electrons in the PPV is more difficult than the injection of holes into the 2-CN-PPV. At this point we may think that the number of holes injected in the PPV single-layer is much higher than the number of electrons injected, being the opposite in the 2-CN-PPV single-layer. Our simulations show that this is not true, and the number of holes and electrons injected in the two single layer structures are almost the same, being the number of charges of a specific sign almost half of the total number of injected charges showed in Fig. 1. The reason for this lies on the fact that the number of charges injected from each electrode depends on the injection of charge of the opposite sign, in the conditions used.

Although we have a non-ohmic contact that can limit the number of charges injected through it, the injection is activated by the applied electric field and the presence of charge of opposite sign in the network. On the other hand, the number of charges injected through the ohmic contact will depend on the space distribution of existing charges in the polymer, which in turn depends on whether charge is collected by the opposite electrode of the injection or whether recombination occurs. For any applied electric field, charge percolation through the polymer layer is limited by the potential barriers between hopping sites, and these barriers depend, among other factors, on the molecular properties of the polymer strands which vary with the strand length [10]. Since the electron affinity varies more significantly with the strand length than the ionization potential, it seems clear that hole percolation in 
PPV network is more difficult than electron percolation in 2-CN-PPV network. As a result, in the 2$\mathrm{CN}-\mathrm{PPV}$ electron transport is achieved in an easier way but is limited by the space distribution of electrons in the polymer. Since the zero-field barrier height in the non-ohmic contact is smaller than the height of the same barrier in the PPV single-layer, the injection of charge of opposite sign is easily achieved so charge recombination occurs earlier reducing the effect of space hole distribution in hole injection. As a result when charge recombination occurs this will allow hole injection, because it will reduce the effect of space charge distribution, and at the same time it will activate electron injection. In other words, in the case of 2-CN-PPV single-layer, electron injection and transport is easier, and recombination occurs earlier, so the number of charges injected will be higher than in the case of PPV single-layer. When the applied electric field increases, the influence of the potential barrier between hopping sites decreases leading to an increase in charge injection and transport through the polymer network. So, when the steady state is achieved, more charge is injected to 2-CN-PPV than to PPV. In the case of the bilayer structure, both electrode/polymer interfaces are ohmic, so injection is limited by space charge distribution which gives rise to an equal number of injected charges as the 2-CN-PPV single-layer structure.

Although the total number of injected charges in the bilayer and 2-CN-PPV single-layer structure seems to be similar, the way that charge undergoes recombination along the axis perpendicular to the electrode surfaces is quite different when the applied electric field is $1 \mathrm{MV} / \mathrm{cm}$. In Fig. 2 we can see that the recombination efficiency is significantly high near the cathode $(0 \mathrm{~nm})$, for the PPV singlelayer, and near the anode $(100 \mathrm{~nm})$, for the 2-CN-PPV single-layer structure. The reason for this lies on the fact that in both single-layer structures the non-ohmic contacts restrict significantly the number of charges injected through them.

In this way, when the injection of a charge through a non-ohmic contact occurs, the probability of that charge undergoing recombination is significantly high, since the number of charges of opposite sign near the electrode also is high. This effect is more pronounced as the zero-field barrier height increases. The presence of the bilayer PPV/2-CN-PPV between the electrodes seems to reduce the recombination efficiency near the electrodes and increase it in the position where the polymer/polymer interface is established. The reason for this behaviour lies on the fact that in this structure both electrode/polymer contacts are ohmic and the polymer/polymer interface seems to increase electron transport in the PPV layer. Fig. 3 shows the fraction of the injected charge that stays stored in the 
bilayer structure for an applied electric field of $1 \mathrm{MV} / \mathrm{cm}$ and the recombination efficiency along the model axis.

Clearly the polymer/polymer interface at $50 \mathrm{~nm}$ distance from both electrodes seems to confine electrons and holes in its neighbourhood, giving rise to an increase in recombination efficiency at this region. A closer look to Fig. 3 indicates that the polymer/polymer interface seems to block preferentially holes rather than electrons and in this way the recombination efficiency in the PPV layer is higher than the recombination efficiency in the 2-CN-PPV, near the interface. At the same time, the number of electrons that remain stored in the PPV layer is higher than the number of holes that stay stored in the 2-CN-PPV. This intriguing behaviour has its origin on that fact that at the polymer/polymer interface the zero-field barrier height for holes is higher than the zero-field barrier height for electrons, due to the difference in the molecular properties of PPV and 2-CN-PPV [10]. Our results show that the effect of the polymer/polymer interface is similar to the one observed in the experiments [11], but in contrast with the experimental results we predict that charge undergoes recombination preferentially in the PPV layer than in the 2-CN-PPV layer. The explanation for this different recombination behaviour may lie on the fact that our model does not contemplate any mechanism for exciton diffusion. Since it is known that the diffusion length of an exciton is around 10 $\mathrm{nm}$, it is reasonable to think that most of the excitons created in the PPV layer will diffuse to the 2$\mathrm{CN}-\mathrm{PPV}$, and then singlet excitons decay radiatively giving rise to light emission on that layer, which is the observed experimental phenomenon.

Fig. 4 shows the internal electric field along the axis perpendicular to the electrodes surface for the structures considered in this work, when the applied electric field is $1 \mathrm{MV} / \mathrm{cm}$. Clearly charge confinement near the polymer/polymer interface leads to a maximum of the internal electric field at that position. At the same time, space charge distribution will lead to an internal electric field profile that allow charge transport towards the polymer/polymer interface and in this way increase the number of charges that undergo recombination at that position. On the other hand, the asymmetry of the internal electric field profile for the PPV and 2-CN-PPV single-layer devices has its origin on the fact that holes/electrons will stay trapped near the electrode/polymer interfaces which will give rise to an increase of the number of charges that undergoes recombination near the electrodes.

Our results show that the effect of the presence of the polymer/polymer interface and the different inter-molecular mobility in the PPV and 2-CN-PPV, due to the difference in the inter-molecular potential barriers for electron and hole hopping in the two polymers, affect the PLED performance at 
an applied electric field of $1 \mathrm{MV} / \mathrm{cm}$. As the applied electric field increases, the effect of the potential barriers between two strands of PPV, 2-CN-PPV, or strands of different polymers tends to vanish, and all three polymer structures tend to have similar profiles for recombination efficiency.

\section{Conclusions}

Although our simulation conditions are ideal, our theoretical results are in agreement with the experimental ones obtained by Greenham et al [11] for a bilayer LED of PPV/CN-PPV. Our results show clearly that the presence of a bilayer as an active component of a PLED can increase its efficiency as compared to the efficiency of its single-layer counterparts, for lower applied electric fields. The presence of the polymer/polymer interface acts as a way to reduce the number of charges that undergo recombination near the electrodes, reducing the exciton quenching effect, and tuning the maximum recombination efficiency zone.

\section{Acknowledgement}

This work is part of the research projects POCTI/CTM/41574/2001 and CONC-REEQ/443/EEI/2005, approved by the Portuguese Foundation for Science and Technology (FCT) and POCTI and support by the European Community Fund FEDER. One of us (H.M.C.B.) is also indebted to FCT for financial support under PhD grant No SFRH/BD/22143/2005.

\section{References}

1. J. H. Burroughes, D. D. C. Bradley, A. R. Brown, R. N. Marks, K. Mackay, R. H. Friend, P. L. Burns and A. B. Holmes, Nature 347539 (1990).

2. E. Moons, Journal of Physics: Condensed Matter 1412235 (2002).

3. M. T. Bernius, M. Inbasekaran, J. O'Brien and W. Wu, Advanced Materials 121750 (2000).

4. A. M. Stoneham, M. M. D. Ramos, A. M. Almeida, H. M. G. Correia, R. M. Ribeiro, H. Ness and A. J. Fisher, Journal of Physics: Condensed Matter 149877 (2002).

5. W. F. Pasveer, J. Cottaar, C. Tanase, R. Coehoorn, P. A. Bobbert, P. W. M. Blom, M. De Leeuw and M. A. J. Michels, Physical Review Letters 941 (2005).

6. M. Pope and C. E. Swenberg, Electronic processes in organic crystals and polymers,Oxford University Press, (1999). 
7. A. M. Almeida, M. M. D. Ramos and H. G. Correia, Computational Materials Science 27128 (2003).

8. B. G. Sumpter, P. Kumar, A. Mehta, M. D. Barnes, W. A. Shelton and R. J. Harrison, Journal of Physical Chemistry B 1097671 (2005).

9.R. M. Ribeiro, M. M. D. Ramos, A. M. Almeida, H. M. G. Correia, J. Silva and A. M. Stoneham, Materials Research Society Symposium - Proceedings, 744549 (2003).

10. M. M. D. Ramos, H. M. G. Correia, R. M. Ribeiro and A. M. Stoneham, Synthetic Metals 147281 (2004).

11. N. C. Greenham, S. C. Moratti, D. D. C. Bradley, R. H. Friend and A. B. Holmes, Nature 365628 (1993).

\section{Figure captions:}

Fig. 1 - The total number of injected charges (electrons and holes) into three polymer networks made of PPV (squares), 2-CN-PPV (circles) and PPV/2-CN-PPV (triangles) as a function of the applied electric field. The lines are just a guide to the eye.

Fig. 2 - Recombination efficiency (proportion of injected electron-hole pairs which recombine) along the model axis (defined as the axis perpendicular to the electrodes surface) for the single-layer structures made of PPV (squares) and 2-CN-PPV (circles), and the PPV/2-CN-PPV (triangles) bilayer structure. The applied electric field is 1 $\mathrm{MV} / \mathrm{cm}$.

Fig. 3 - Proportion of injected charges (electrons and holes) that are trapped or in transit within the 2-CN-PPV/PPV bilayer network in the steady state, and the recombination efficiency along the axis perpendicular to the electrodes surface in PPV (triangles) and 2CN-PPV (squares) layers, for an applied electric field of $1 \mathrm{MV} / \mathrm{cm}$.

Fig. 4 - The internal electric field along the model axis perpendicular to the electrodes surface for PPV (squares), 2-CN-PPV (circles) and PPV/2-CN-PPV bilayer (triangles) structures. These results were obtained using an applied electric field of $1 \mathrm{MV} / \mathrm{cm}$ which is represented by the dotted line. 
Fig.1

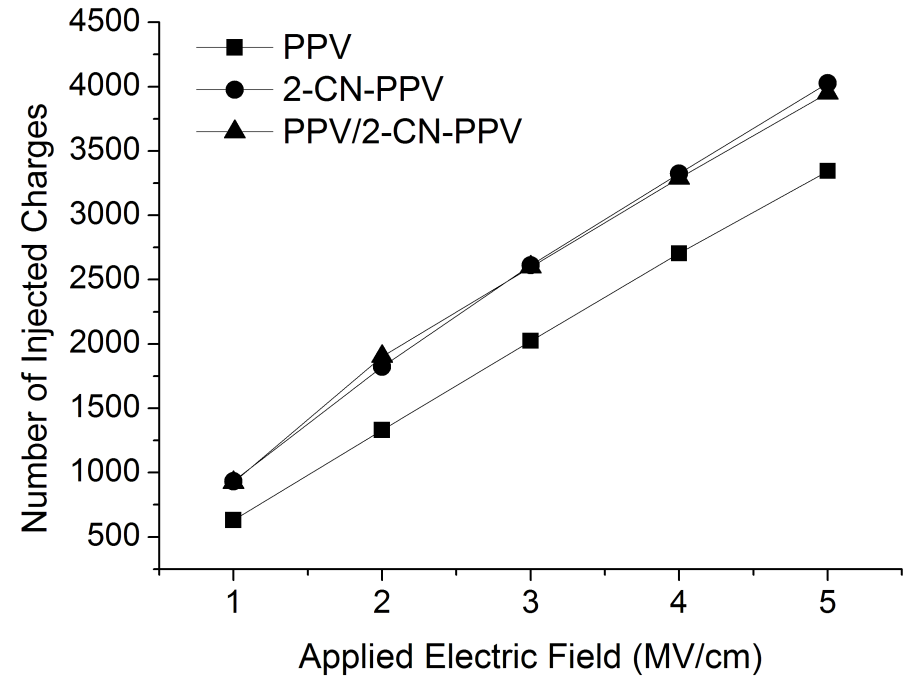


Fig. 2

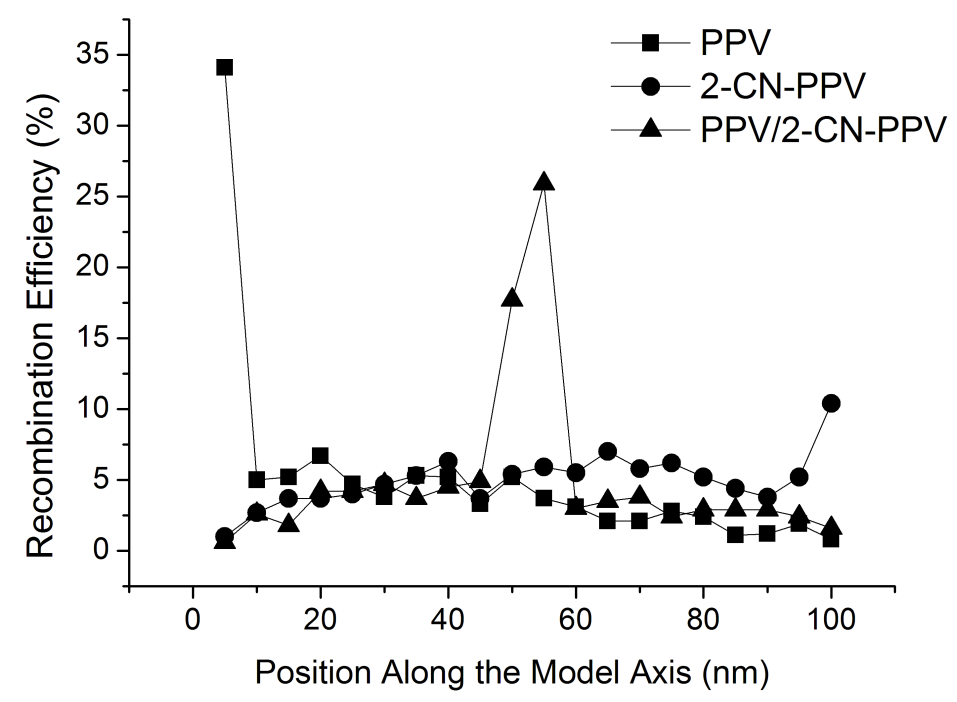


Fig. 3

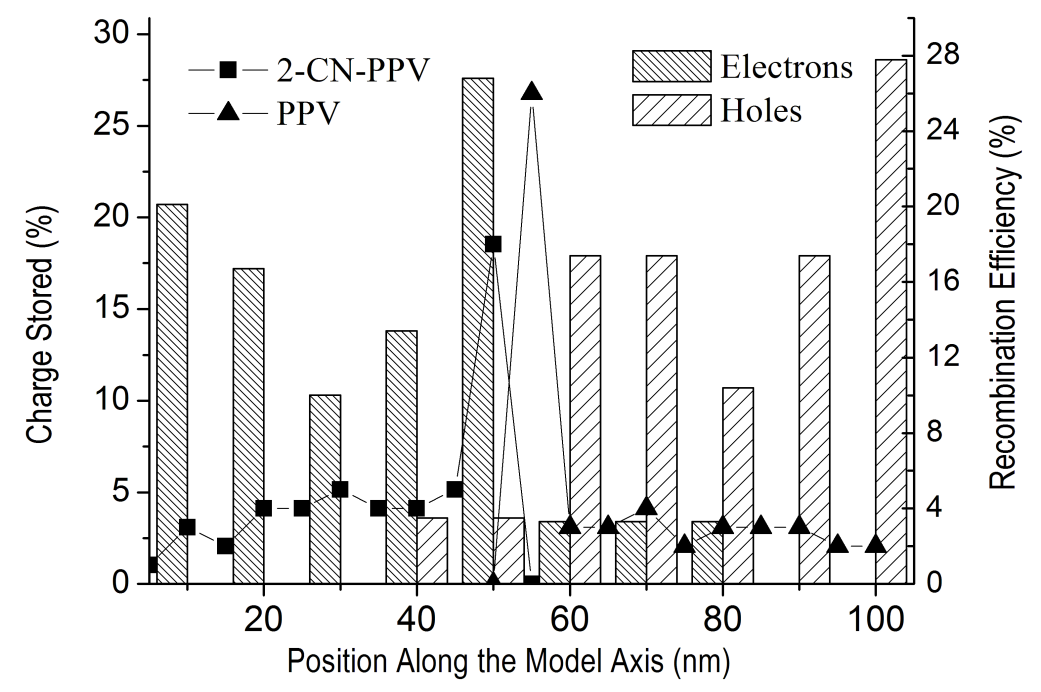


Fig. 4

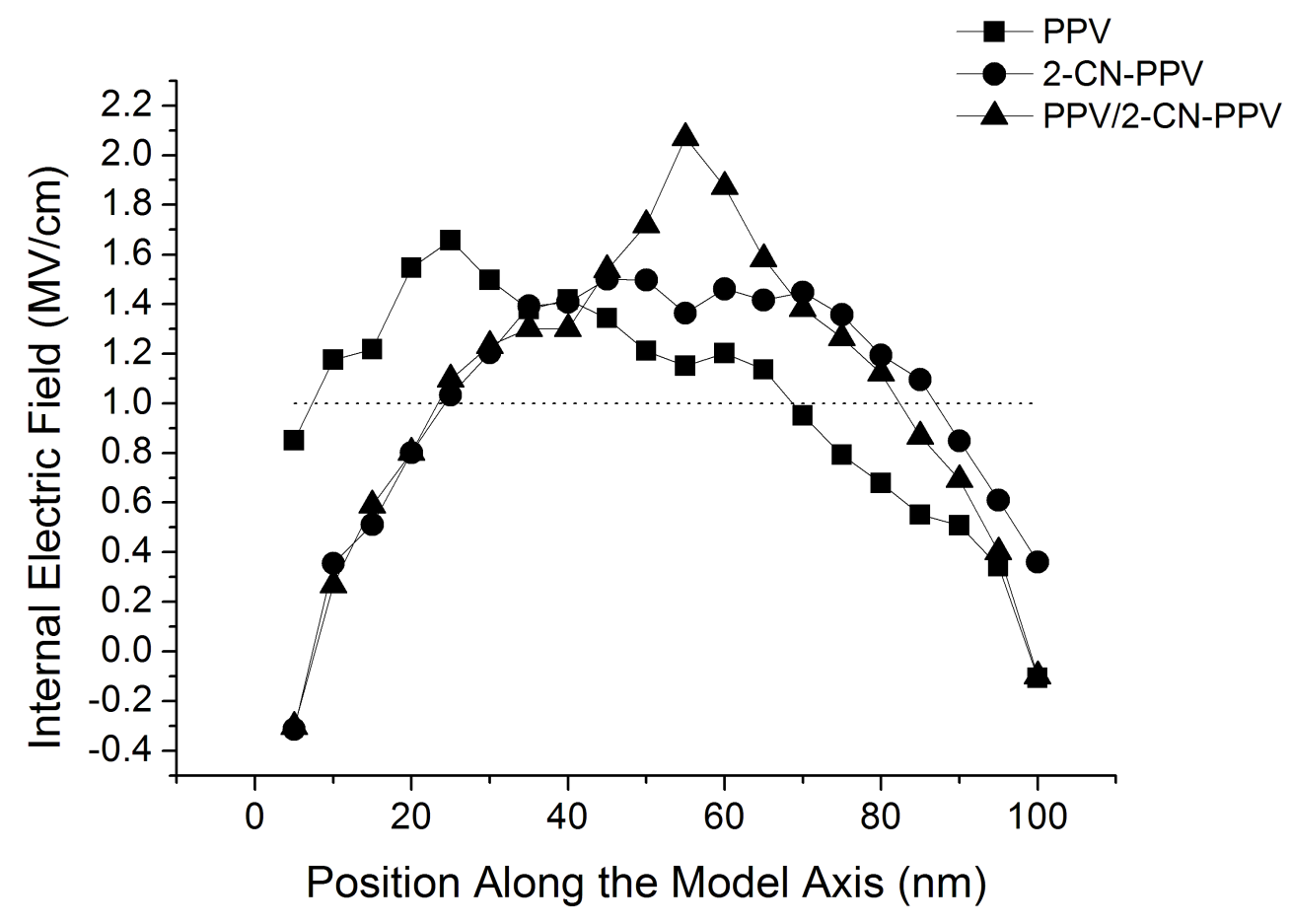

